\title{
Regulatory Dendritic Cells Induced by Mesenchymal Stem Cells Ameliorate Dextran Sodium Sulfate-Induced Chronic Colitis in Mice
}

\author{
Hannah Jo ${ }^{1}$, Young Woo Eom ${ }^{2}$, Hyun-Soo Kim ${ }^{1}$, Hong Jun Park ${ }^{1}$, Hee Man Kim ${ }^{1}$, and Mee-Yon Cho ${ }^{3}$ \\ ${ }^{1}$ Department of Internal Medicine, ${ }^{2}$ Cell Therapy and Tissue Engineering Center, and ${ }^{3}$ Department of Pathology, Yonsei University Wonju \\ College of Medicine, Wonju, Korea
}

Background/Aims: Regulatory dendritic cells (rDCs), which can be induced by mesenchymal stem cells (MSCs), play an important role in inducing and maintaining homeostasis of regulatory $T$ cells and exhibit anti-inflammatory functions. In this study, we investigated whether MSCs could differentiate DCs into rDCs and compared the therapeutic effects of rDCs and MSCs on dextran sodium sulfate (DSS)-induced chronic colitis mice. Methods: Immature DCs (imDCs) and lipopolysaccharide (LPS)-treated mature DCs (mDCs) were co-cultured with MSCs for 48 hours, and then the profiles of surface markers and cytokines and regulatory roles of these DCs for primary splenocytes were analyzed. In addition, the therapeutic effects of MSCs and DCs co-cultured with MSCs were compared in chronic colitis mice. Results: After co-culture of imDCs (MSC-DCs) or LPS-treated mDCs (LPS+MSCDCs) with MSCs, the expression of CD11c, CD80, CD86, interleukin 6 (IL-6), tumor necrosis factor- $\alpha$ (TNF- $\alpha$ ), and interferon- $\gamma$ (IFN- $\gamma$ ), was decreased, but that of CD11b, IL10 , and transforming growth factor- $\beta$ (TGF- $\beta$ ) was increased. Furthermore, MSC-DCs and LPS+MSC-DCs induced the expression of CD4, CD25, and Foxp3 in primary splenocytes isolated from mice. In DSS-induced colitis mice, MSCs and MSC-DCs increased colon length, body weight, and survival rate and induced histological improvement. Moreover, in the colon tissues, the expression of IL- 6 , TNF- $\alpha$, and IFN- $\gamma$ decreased, but that of IL-10, TGF- $\beta$, and Foxp3 increased in the MSC- and MSC-DC-injected groups. Conclusions: Our data suggest that MSCs differentiate DCs into rDCs, which ameliorate chronic colitis. Thus, rDCs stimulated by MSCs may be therapeutically useful for the treatment of chronic inflammatory diseases. (Gut Liver 2018;12:664-673)

Key Words: Inflammatory bowel diseases; Mesenchymal stromal cells; Dendritic cells; T-lymphocytes, regulatory; Antiinflammatory effect

\section{INTRODUCTION}

Inflammatory bowel disease (IBD), characterized by chronic and relapsing intestinal inflammation, has become a major health care concern worldwide due to a steady increase in incidence and prevalence. ${ }^{1}$ The etiology of IBD is largely unknown. The latest evidence regarding its pathogenesis suggests that IBD is related to inappropriate and overstimulated immune responses, especially abnormal $\mathrm{T}$ cell responses to the normal gut flora and innocuous food antigens, which can be caused by genetic susceptibility. ${ }^{2}$ Recent studies have suggested that mesenchymal stem cells (MSCs), which have self-renewal and immunosuppressive capacities, exhibit therapeutic functions in the treatment of IBD. ${ }^{3-5}$ MSCs have been reported to express indoleamine 2,3-dioxygenase (IDO) and inducible nitric oxide synthase (iNOS) or secrete various molecules, including transforming growth factor (TGF)- $\beta$, prostaglandin E2 (PGE2), and interleukin (IL)-10. ${ }^{6}$ All these molecules are well-known to play immunosuppressive roles. In particular, T-lymphocyte activities are suppressed by the paracrine effects of MSCs; these facts provide a rationale for potentially utilizing MSCs in the clinical treatment of autoimmune diseases. ${ }^{7,8}$ Additionally, the immunosuppressive functions of MSCs have been shown to be effective in several animal models of IBD induced by 2,4,6-trinitro benzene sulfonic acid or dextran sodium sulfate (DSS). ${ }^{9}$ However, the mechanisms by which MSCs suppress T-cell proliferation, and how to harness these mechanisms for the treatment of IBD, remain unclear.

It has also been reported that MSCs can modulate the phenotype of dendritic cells (DCs) because of their immunosuppressive roles. ${ }^{11-13}$ DCs are crucial antigen-presenting cells that mediate

Correspondence to: Hyun-Soo Kim

Department of Internal Medicine, Yonsei University Wonju College of Medicine, 20 Ilsan-ro, Wonju 26426, Korea

Tel: +82-33-741-1224, Fax: +82-33-747-3538, E-mail: hyskim@yonsei.ac.kr

Received on February 1, 2018. Revised on April 11, 2018. Accepted on April 23, 2018. Published online September 21, 2018

pISSN 1976-2283 eISSN 2005-1212 https://doi.org/10.5009/gnl18072

Hannah Jo and Young Woo Eom contributed equally to this work as first authors.

@) This is an Open Access article distributed under the terms of the Creative Commons Attribution Non-Commercial License (http://creativecommons.org/licenses/by-nc/4.0) which permits unrestricted non-commercial use, distribution, and reproduction in any medium, provided the original work is properly cited. 
both innate and adaptive immune responses. ${ }^{14}$ Once DCs confront and capture antigens, they migrate to a lymphoid organ where they interact with naïve $\mathrm{T}$ cells and $\mathrm{B}$ cells, and begin to promote T-cell immune responses. ${ }^{15}$ This leads to the activation of antigen-specific $\mathrm{T}$ cells, eventually resulting in inflammation. If the encountered antigens are found to be innocuous in the course of the inflammatory response, DCs may begin to exhibit a tolerogenic phenotype, suggesting their tolerogenic potential during this stage of immune response. Therefore, mucosal DCs can be regulated by the microenvironment. ${ }^{16,17}$ The immunomodulatory role of DCs has been highlighted in IBD, and is thought to be caused by dysregulation of inflammatory responses. ${ }^{18,19}$ Thus, we postulate that the interaction between MSCs and DCs may play a potential therapeutic role in the treatment of IBD, which can be attributed to the immunosuppressive functions of MSCs. MSCs can inhibit both stages of DC maturation: the differentiation stage from precursor DCs to immature DCs (imDCs) ${ }^{20}$ and the differentiation stage from imDCs to mature DCs (mDCs). ${ }^{21}$ Once DCs receive anti-inflammatory signals, they secrete molecules such as TGF- $\beta$, IL-10, and PGE ${ }_{2}$, and are often stimulated to turn tolerogenic. These regulatory DCs (rDCs) further stimulate the production of regulatory T cells (Tregs). ${ }^{12}$ Given that MSCs can alter the function of DCs, rDCs modulated by MSCs should also exert an anti-inflammatory effect on immune disorders. ${ }^{13,22,23}$

In this study, we found that DCs co-cultured with MSCs are effectively differentiated into rDCs, which further attenuate DSS-induced chronic colitis in mice through the induction of Tregs, similar to MSCs. More importantly, we demonstrated that MSCs convert both imDCs and mDCs into rDCs, both in vivo and in vitro, resulting in improvement of chronic colitis.

\section{MATERIALS AND METHODS}

\section{Mice}

Female C57BL/6 mice (5- to 8-week-old) were purchased from Orient Bio, Inc. (Seongnam, Korea). Animals were maintained in a 12-hour light/dark cycle under standard conditions at $25^{\circ} \mathrm{C}$. Throughout the experiment, animals were given food and water ad libitum. All animal experiments were approved by the Institutional Animal Care and Use Committee of Yonsei University, Wonju College of Medicine.

\section{Induction of chronic colitis and transplantation of cells}

DSS (Molecular weight 36-50 kDa; MP Biomedicals, Santa Ana, CA, USA) was dissolved in drinking water at a $3 \%$ concentration (w/v) and given to a group of C57BL/6 mice. Fresh DSS solutions were prepared daily. To induce chronic colitis, the mice underwent two cycles of DSS treatment. One cycle consisted of 5 days of DSS-treated water, followed by 5 days of normal drinking water. imDCs, MSCs, and MSC-DCs $\left(1 \times 10^{6}\right.$ cells $)$ were administered by two intraperitoneal injections on days 6 and 16. The animals were observed daily for changes in body weight, diarrhea, and bleeding. Animal weights were monitored and recorded daily.

\section{Histology}

Mice were sacrificed by cervical dislocation and the whole colon was removed immediately up to the cecum. The colon $(1.5 \mathrm{~cm})$ was fixed in 10\% formalin, embedded in paraffin, and sectioned at a 4 to $5 \mu \mathrm{m}$ thickness with a microtome. Sections were stained with hematoxylin \& eosin to analyze the degree of inflammation. Histological changes were assessed according to the criteria previously described by Rayudu and Raju. ${ }^{24}$

\section{Isolation, culture, and characterization of MSCs}

MSCs were isolated and cultured from the compact bone of mouse femurs and tibias as described previously. ${ }^{25}$ MSCs were plated at a concentration of $10^{6}$ cells/mL in $\alpha$-Minimum Essential Medium Eagle (Hyclone Laboratories, Pittsburgh, PA, USA) supplemented with 10\% fetal bovine serum (FBS; Hyclone Laboratories) and $1 \%$ penicillin/streptomycin at $37^{\circ} \mathrm{C}$ in a $5 \% \mathrm{CO}_{2}$ humidified atmosphere. At $80 \%$ to $90 \%$ confluence, MSCs were treated with 0.25\% trypsin/EDTA (Sigma-Aldrich, San Diego, CA, USA) for 4 minutes, and diluted at a ratio of $1: 2$ per passage. MSCs were used in experiments after 3 to 5 passages, to ensure depletion of monocytes/macrophages. MSCs were characterized by flow cytometry for the expression of the common MSC markers, CD29 and CD105, the progenitor cell marker stem cell antigen (Sca)-1, the epithelial cell marker CD31, and for the absence of the hematopoietic cell markers, CD45 and CD11b. All antibodies for detection of MSC markers were purchased from eBioscience (San Diego, CA, USA). All data from flow cytometry analyses (FACSAria III; BD Biosciences, San Jose, CA, USA) were expressed as percentages.

\section{Culture, generation, and characterization of DCs}

DCs were isolated and cultured from the mouse femur and tibia bone marrow. During cell isolation, $5 \mathrm{~mL}$ of red blood cell lysis buffer (Sigma-Aldrich) was added to the cell suspension. After lysis at room temperature for 5 minutes, cells were centrifuged at 1,500 rpm for 5 minutes. To obtain imDCs, cells were cultured in a culture dish containing RPMI 1640 media supplemented with 10\% FBS (Hyclone Laboratories), 1\% penicillin/ streptomycin, recombinant mouse GM-CSF (40 ng/mL; JW CreaGene, Seongnam, Korea), and IL-4 (40 ng/mL; JW CreaGene) for 7 days, following which imDCs were treated with lipopolysaccharide (LPS; $200 \mathrm{ng} / \mathrm{mL}$; Sigma-Aldrich) for 48 hours for maturation (LPS-DCs). The phenotypes of imDCs and LPS-DCs were analyzed using a flow cytometer (FACSAria III, BD Biosciences).

\section{Co-culture of MSCs and DCs}

A Transwell ${ }^{\circledR}$ system $(0.8-\mu m$ pore size membrane; SPL Life 
Sciences, Pocheon, Korea) was used to prevent the MSCs from achieving direct contact with the DCs. MSCs and imDCs were seeded into the upper and lower layers respectively, of the Transwell ${ }^{\circledR}$ plate at a ratio of 1:5. In the co-culture of imDCs and MSCs, imDCs treated with or without LPS were labeled as LPS+MSC-DCs or MSC-DCs, respectively.

\section{Co-culture of splenocytes and MSC-DCs}

Whole spleens were isolated from healthy female 5-week-old C57BL/6 mice. A Transwell ${ }^{\circledR}$ system $(0.8-\mu m$ pore size membrane; SPL Life Sciences) was used to prevent MSC-DCs from achieving direct contact with the splenocytes. After 2 days of co-culture of MSCs and imDCs with or without LPS, only the MSC-DCs or LPS+MSC-DCs cultured in the lower chamber were collected, and along with the splenocytes were re-seeded at a ratio of 1:5 into the upper and lower layers, respectively, of the Transwell ${ }^{\circledR}$ plate, and further cultured for 48 hours.

\section{Flow cytometry analysis}

For the analysis of cell surface marker expression of MSCs or DCs, phycoerythrin (PE)-labeled anti-CD11b, -CD31, and -CD105; fluorescein isothiocyanate (FITC)-labeled anti-CD45, -CD29, and -Sca-1 (eBioscience) or allophycocyanin (APC)labeled anti-CD11c, -CD80, and -CD86 (BioLegend, San Diego, CA, USA) were used, respectively.

\section{Reverse transcription-polymerase chain reaction}

Total RNA from tissues and cells was isolated using TRIzol reagent (Sigma-Aldrich). cDNA was prepared using $1 \mu \mathrm{g}$ of total RNA from each sample, according to the manufacturer's protocol (Toyobo, Tokyo, Japan). For endpoint quantitative polymerase chain reaction (PCR), a PCR master mix (Elpis Biotech, Daejeon, Korea) was used. The primers used in this study are listed in Table 1.

\section{Western blotting}

Mouse colons and cells were homogenized in PRO-PREP ${ }^{\mathrm{TM}}$ protein extraction solution (iNtRON Biotechnology, Seongnam, Korea) with proteinase and phosphatase inhibitors (GenDEPOT, Barker, TX, USA). The concentration of the supernatant protein was measured using the bicinchoninic acid protein assay kit (Pierce, Rockford, IL, USA). Proteins were then separated by sodium dodecyl sulfate-polyacrylamide gel electrophoresis (8\% to $12 \%$ ) and transferred to a polyvinylidene difluoride membrane. The blots were incubated with the following primary antibodies: anti-IL-10, anti-Foxp3, anti-TGF- $\beta$, anti-STAT3, anti-pSTAT3, and anti- $\beta$-actin (Abcam, Cambridge, MA, USA). Blots were visualized using a chemiluminescence UVP BioSpectrum 600 imaging system (Upland, CA, USA).

\section{Immunohistochemistry}

For immunohistochemical staining, deparaffinized colon sections were incubated with rabbit anti-Foxp3 primary antibody (Abcam). After that, sections were incubated with rabbit antiIgG secondary antibody (Abcam) for 30 minutes using the antibody enhancer horseradish peroxidase polymer (Biocare Medical). Slides were counterstained with hematoxylin. To assess Foxp $^{+}$cells, digital images at $\times 200$ and $\times 400$ magnification were obtained (Zeiss Axio Imager M1, Göttingen, Germany). Both positive cells and total cells were counted from three random fields, with over 200 cells counted per field.

\section{Statistical analysis}

Data were expressed as the mean \pm standard error of the mean using a one-way analysis of variance (Scheffe test). Any difference was considered to be statistically significant at $\mathrm{p}<0.05$.

Table 1. Primers Used for Polymerase Chain Reaction Reactions

\begin{tabular}{lll}
\hline \multicolumn{1}{c}{ Gene } & \multicolumn{1}{c}{ Forward (5'-3') } & \multicolumn{1}{c}{ Reverse (5'-3') } \\
\hline$\beta$-Actin & GTGGGCCGCCCTAGGCACCAG & CTCTTTGATGTCACGCACGATTTC \\
IL-6 & AGGTCTGTTGGGAGTGGTATC & AGGTCTGTTGGGAGTGGTATC \\
TNF- $\alpha$ & CATCTTCTCAAAATTCGAGTGACAA & TGGGAGTAGACAAGGTACAACCC \\
IFN- $\gamma$ & TCAAGTGGCATAGATGTGGAAGAA & TGGCTCTGCAGGATTTCATG \\
IL-10 & GCCAAGCCTTATCGGAAATG & CACCCAGGGAATTCAAATGC \\
TGF- $\beta$ & AACAATTCCTGGCGTACCTT & CTGCCGTACAACTCCAGTGA \\
Foxp3 & TTGGCCAGCGCCATCTT & TGCCTCCTCCAGAGAGAAGTG \\
CD4 & GAGAGTCAGCGGAGTTCTC & CTCACAGGTCAAAGTATTGTTG \\
CD8 & GCTCAGTCATCAGCAACTCG & ATCACAGGCGAAGTCCAATC \\
CD25 & CTCCCATGACAAATCGAGAAAGC & ACTCTGTCCTTCCACGAAATGAT \\
\hline
\end{tabular}

IL, interleukin; TNF, tumor necrosis factor; IFN, interferon; TGF, transforming growth factor. 


\section{RESULTS}

\section{MSCs modulate the phenotypes of DCs and stimulate Tregs}

MSCs isolated from mice were positive for CD29, CD105, and Sca-1, but negative for CD45, CD11b, and CD31 (Fig. 1). The phenotype of MSCs was similar to the previously reported typical MSC phenotype. ${ }^{26}$ The phenotype of imDCs and LPSDCs was CD $11 \mathrm{c}^{+} \mathrm{CD}^{\text {low }} \mathrm{CD} 86^{\text {low }}$ and $\mathrm{CD} 11 \mathrm{c}^{+} \mathrm{CD} 80^{\text {high }} \mathrm{CD} 86^{\text {high }}$, respectively. When imDCs and MSCs were co-cultured with or without LPS, CD11b expression was increased in both MSCDCs and LPS+MSC-DCs, but the expression of CD80 and CD86 was decreased compared to LPS-DCs. In addition, the expression of CD11c was remarkably decreased in both MSC-DCs and LPS + MSC-DCs (Fig. 2A). LPS-DCs highly expressed inflammatory cytokines such as IL-6, tumor necrosis factor (TNF)- $\alpha$, and interferon (IFN)- $\gamma$. However, after co-culturing imDCs and MSCs with or without LPS, the expression of these cytokines disappeared, but the expression of IL-10 and TGF- $\beta$ markedly increased (Fig. 2B). These results suggest that MSCs induce both imDCs and LPS-DCs into rDCs, which express anti-inflammatory cytokines, such as IL-10 and TGF- $\beta$. To investigate whether rDCs (MSC-DCs and LPS+MSC-DCs) stimulate the production of Tregs, four kinds of DCs, including imDCs, LPS-DCs, MSC-DCs, and LPS+MSC-DCs were co-cultured with splenocytes, and then the markers of Tregs (i.e., Foxp3, CD4, and CD25) were analyzed by reverse transcription-polymerase chain reaction and immunoblotting. After 2 days of co-culture, in splenocytes cocultured with MSC-DCs or LPS + MSC-DCs, the mRNA expression of Foxp3, CD4, and CD25 was significantly increased (Fig. 3A). Foxp3 protein expression also markedly increased both in splenocytes co-cultured with MSC-DCs or LPS+MSC-DCs (Fig. 3B). Moreover, the expression levels of Foxp3, CD4, and CD25 were the highest in splenocytes co-cultured with LPS+MSC-DCs (Fig. 3). These results suggest that DCs co-cultured with MSCs can stimulate the production of Tregs by functioning as rDCs.

\section{MSC-DCs ameliorate DSS-induced chronic colitis}

In order to prevent abnormal results from the inflammatory response caused by the small amount of residual LPS, LPS-DCs and LPS+MSC-DCs were excluded from the experimental groups of chronic colitis mice. To evaluate whether rDCs triggered by MSCs can ameliorate chronic colitis and whether their therapeutic effects are higher than that of MSC transplantation, cells (i.e., imDCs, MSC-DCs, and MSCs) were injected intraperitoneally into DSS-induced chronic colitis mice twice, at 6 and 16 days. As a result, 57\% of the DSS-induced chronic colitis mice died within 20 days, and the survival rates were further decreased by imDC injection. Interestingly, all mice injected with MSCs and MSC-DCs survived until the end of experiments (Fig. 4A). During the experiment, the body weight of imDC-transplanted mice gradually decreased, similar to that of saline mice, while the body weight of MSC- and MSC-DC-transplanted mice continued to increase. In particular, the body weight of MSC-DCs group increased to almost the same level as that of control group at 20 days (Fig. 4B). The colon length was also significantly recovered in MSC- and MSC-DC-transplanted groups, but shortening of the colon was observed in both saline-treated chronic colitis and imDC-treated groups (Fig. 4C). In order to determine the histological improvements after injection of MSCs and MSCDCs, randomly selected tissue slides were graded according to the degree of epithelial damage, loss of crypts, and infiltration of inflammatory cells (Table 2). The MSCs and MSC-DCs groups had relatively lower scores (4.66 and 4.33, respectively) than the saline-treated chronic colitis group $(7.66 ; n=3)$ and imDC-injected group (7.33) (Fig. 4D). These results suggest that both MSCs and MSC-DCs ameliorate chronic colitis, implying that there is a possible treatment for colitis using MSC-DCs stimulated by MSCs in vitro.

\section{MSC-DCs ameliorate chronic colitis by modulation of in- flammatory cytokines}

To confirm whether the therapeutic effects of MSCs and MSC-

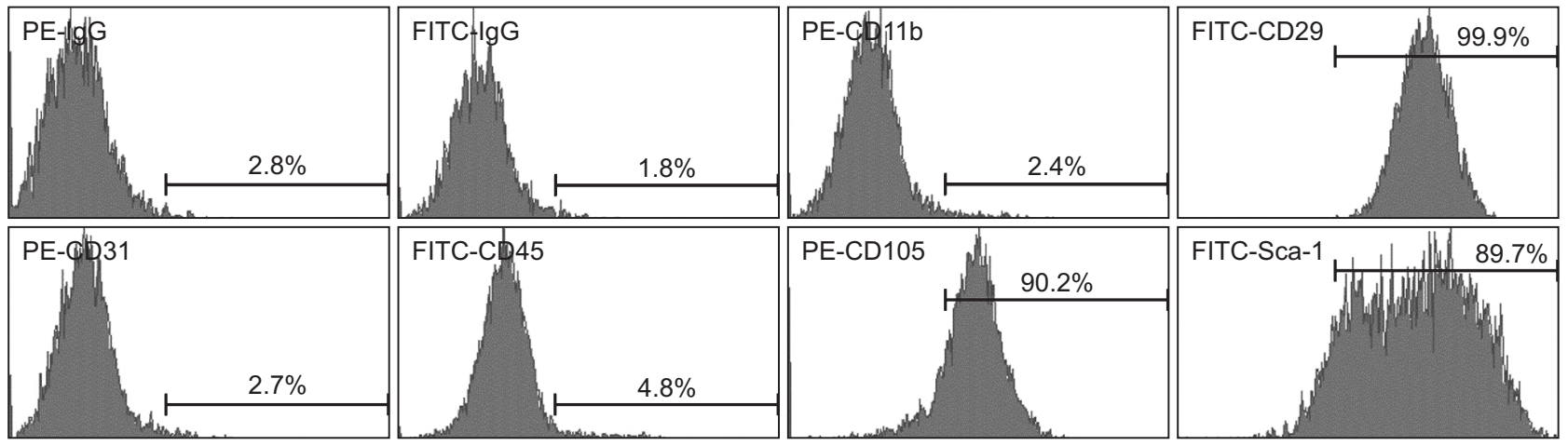

Fig. 1. The surface markers of mesenchymal stem cells (MSCs) were analyzed by flow cytometry. MSCs were negative for CD45 and CD11b (hematopoietic cell markers) and CD31 (endothelial cell marker). MSCs were positive for Sca-1 (progenitor cell marker) and CD105 and CD29. PE, phycoerythrin; FITC, fluorescein isothiocyanate; Sca, stem cell antigen. 
A
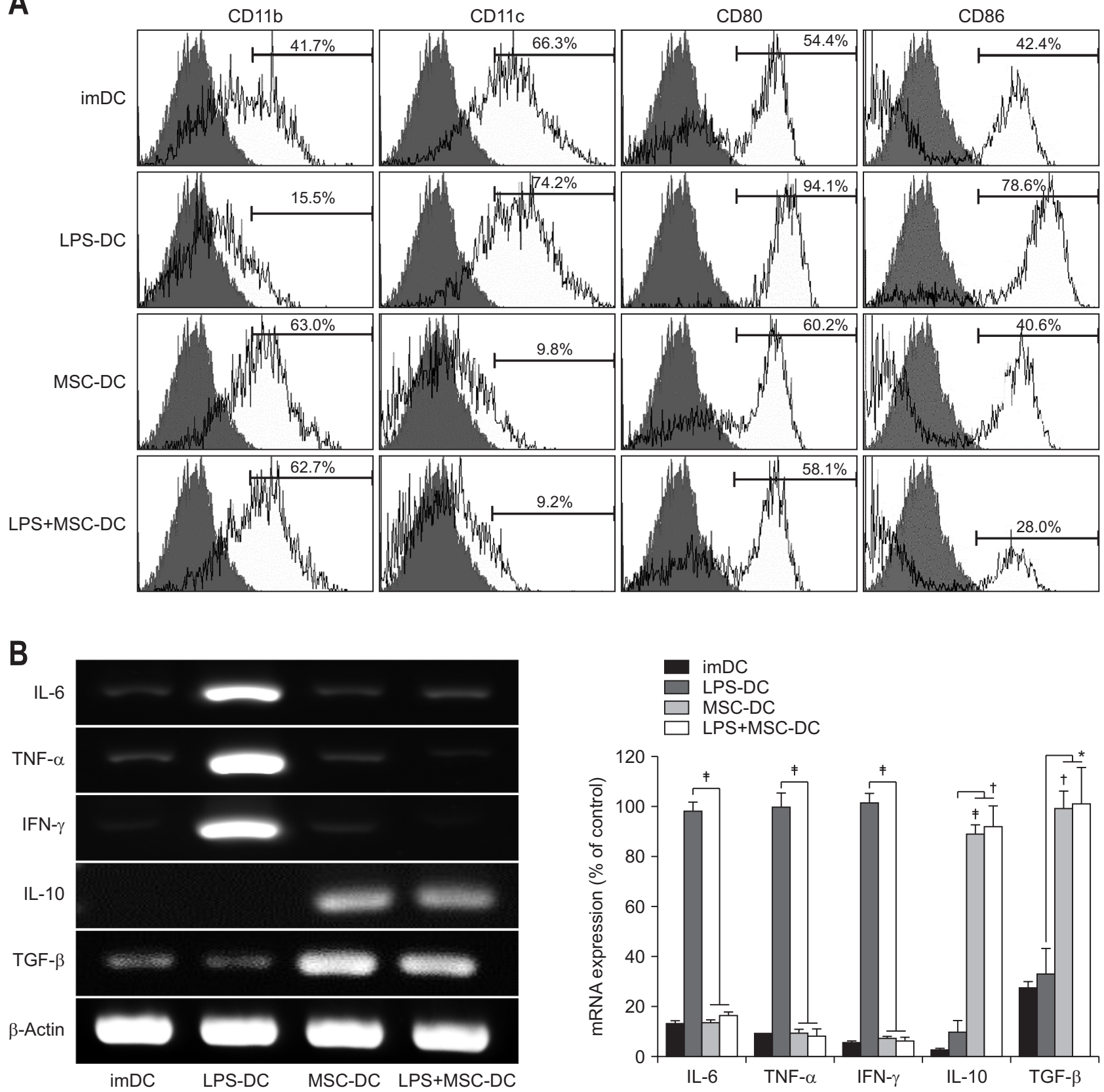

Fig. 2. Mesenchymal stem cells (MSCs) induce immature dendritic cells (imDCs) and lipopolysaccharide (LPS)-DCs into a distinct DC subset. LPSDCs and imDCs were co-cultured with MSCs with or without LPS for 2 days. (A) Surface markers (i.e., CD11b, CD11c, CD80, and CD86) were analyzed by flow cytometry. (B) The expression of pro- (IL-6, TNF- $\alpha$, and IFN- $\gamma$ ) and anti-inflammatory (IL-10 and TGF- $\beta$ ) cytokines was detected in four kinds of DCs (i.e., imDCs, LPS-DCs, MSC-DCs, and LPS+MSC-DCs) by reverse transcription-polymerase chain reaction, and then, relative mRNA expression was analyzed by densitometry to compare the expression levels (LPS-DCs vs MSC-DCs groups and LPS-DCs vs LPS+MSC-DCs groups). Statistical analysis was performed using a one-way analysis of variance. $\beta$-Actin was used as a loading control. Data are expressed as the mean \pm standard error of the mean in triplicate experiments.

IL, interleukin; TNF, tumor necrosis factor; IFN, interferon; TGF, transforming growth factor. ${ }^{*} \mathrm{p}<0.05,{ }^{\dagger} \mathrm{p}<0.001$, and ${ }^{\ddagger} \mathrm{p}<0.0001$.

DCs observed in Fig. 4 were associated with changes in pro- or anti-inflammatory cytokine profiles, these cytokines were analyzed in the colon tissues obtained from mice injected with cells (i.e., imDCs, MSCs, MSC-DCs) or left untreated (i.e., control and saline). Pro-inflammatory cytokines, such as IL-6, TNF- $\alpha$, IFN- $\gamma$ were increased in saline or imDC-injected groups (Fig. 5A).
However, similar to in vitro data, MSC-DCs showed reduced expression of pro-inflammatory cytokines, but significantly increased expression of anti-inflammatory cytokines (i.e., IL-10 and TGF- $\beta$ ). Similar results were also observed in MSC-injected colon tissues (Fig. 5A). We also observed that the protein levels of IL-10 and TGF- $\beta$ increased in both MSC- and MSC-DC-in- 
jected groups. In addition, phosphorylation of STAT3, a downstream molecule of IL-6, was dramatically suppressed in both MSC- and MSC-DC-injected groups, but was increased in saline and imDC-injected groups (Fig. 5B). These results suggested that the therapeutic effects of MSCs and MSC-DCs may be associated with changes in pro- or anti-inflammatory cytokine profiles and that both cell types might share the same therapeutic pathway.

\section{MSC-DCs and MSCs increase Tregs in vivo}

To observe whether MSC-DCs and MSCs stimulate Treg activation in colitis model, the number of Foxp $3^{+}$cells and expression levels of Foxp3 were evaluated. After transplantation of imDCs, MSC-DCs, and MSCs, Foxp3 ${ }^{+}$cells were assessed by immunohistochemical staining of the colon tissues. As shown in Fig. 5A, Foxp3 ${ }^{+}$and other immune cells were rarely observed in the colon tissues of the normal (saline- and imDC-injected) groups, but many immune cells were recruited in the colon of the MSC- and MSC-DC-injected groups, and about 20\% Foxp3 ${ }^{+}$ cells were observed (Fig. 6A). Similar to the results of immunohistochemical staining, Foxp3 protein expression was increased in MSC- and MSC-DC-injected groups (Fig. 6B). These results suggest that MSCs and MSC-DCs can also activate Tregs in vivo, and these Tregs may serve as therapeutic targets to treat chronic colitis. In addition, these results also suggest that rDCs may potentially be used in the treatment of inflammatory diseases, such as IBD, similar to MSCs, which are utilized for various immunosuppressive treatments in vivo and in vitro.

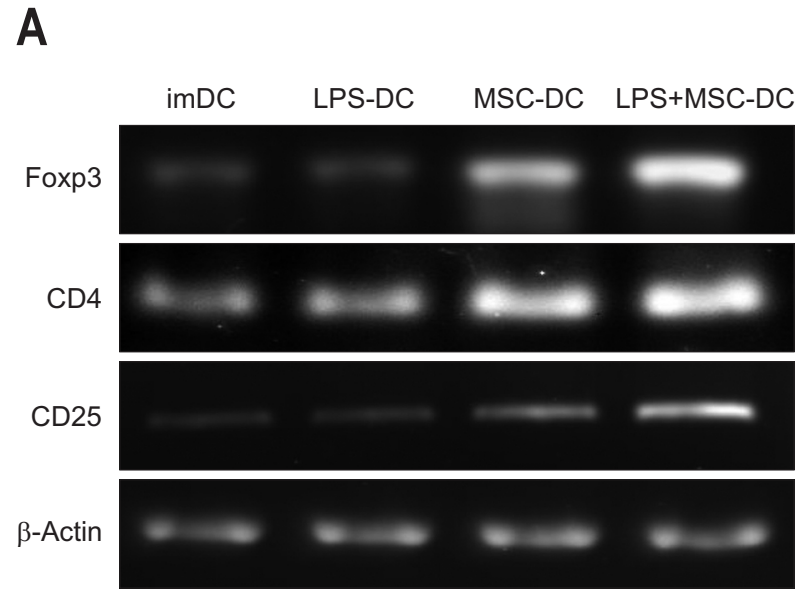

\section{DISCUSSION}

MSCs have been shown to inhibit systemic responses to various lymphocytes, and this function has been implicated in the treatment of IBD. When DCs are stimulated by MSCs, they lose their typical immune-stimulatory function and assume an anti-inflammatory role instead. ${ }^{27}$ One of the mechanisms for this phenotypic change was elucidated by recent studies, which showed that DCs may be affected by the secretion of IL-10 and TGF- $\beta$ by MSCs, and that MSCs may promote the anti-inflammatory activity of DCs both through inhibition of DC maturation and promotion of phenotypic changes in Tcells. $^{21,27,28}$ Moreover, MSC-DCs produce less IL-12, but more IL10 and TGF- $\beta$, a characteristic of the rDC population, ${ }^{13,29}$ similar to our results. In the present study, when DCs were co-cultured with MSCs, we found that the expression of the inflammationrelated co-stimulatory cell surface markers, CD80 and CD86, was significantly decreased. Furthermore, MSC-DCs significantly increased the expression of anti-inflammatory cytokines (IL-10 and TGF- $\beta$ ), reduced the expression of pro-inflammatory cytokines (IL-6, TNF- $\alpha$, and IFN- $\gamma$ ), and stimulated the splenocytes to produce the markers of Tregs (Foxp3, CD4, and CD25). Tregs are well-known and key suppressors of inflammation. In addition, the differentiation signals of these cells have been documented, and TGF- $\beta$ and IL-10 have been identified as the key cytokines for immune suppression. ${ }^{30,31}$

In chronic colitis, MSC-DCs increased the survival rate, body weight, colon length, and histological improvement, similar to MSCs. In addition, both MSCs and MSC-DCs commonly inhibited the expression of pro-inflammatory cytokines (IL-6, TNF- $\alpha$,

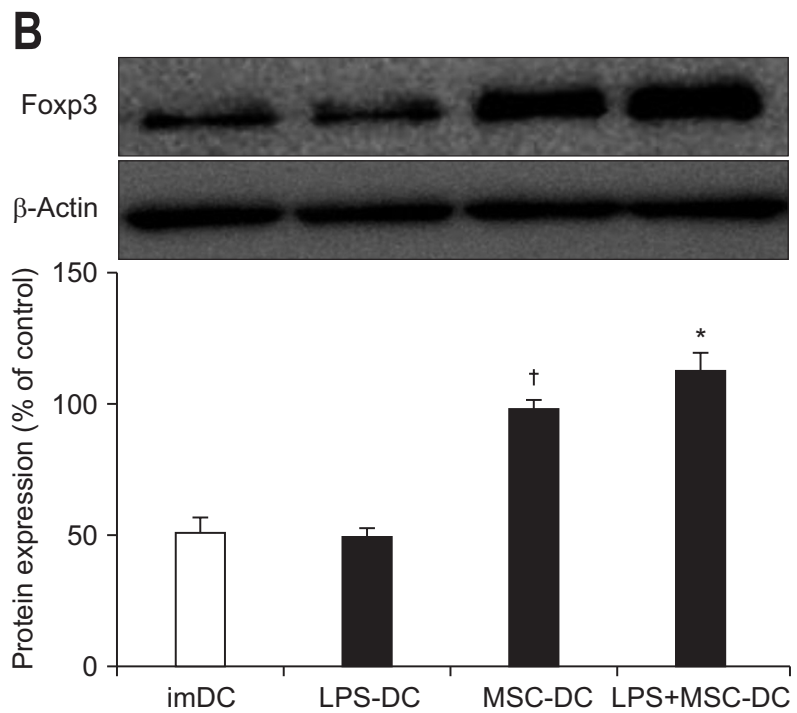

Fig. 3. MSC-DCs induce differentiation of splenocytes into regulatory T cells (Tregs). The spleens were isolated from healthy and young C57BL/6 female mice. Naïve T-cell-enriched splenocytes were co-cultured with each group for 48 hours (imDCs, LPS-DCs, MSC-DCs, or LPS+MSCDCs:splenocytes=1:5). CD4, CD25, and Foxp3 were used as markers of Tregs, and their mRNA levels were detected by reverse transcription-polymerase chain reaction (A). The levels of Foxp3 protein were assessed by Western blotting (B). MSCs, mesenchymal stem cells; DCs, dendritic cells; imDC, immature DCs; LPS, lipopolysaccharide. ${ }^{*} \mathrm{p}<0.001$, and ${ }^{\dagger} \mathrm{p}<0.0001$. 

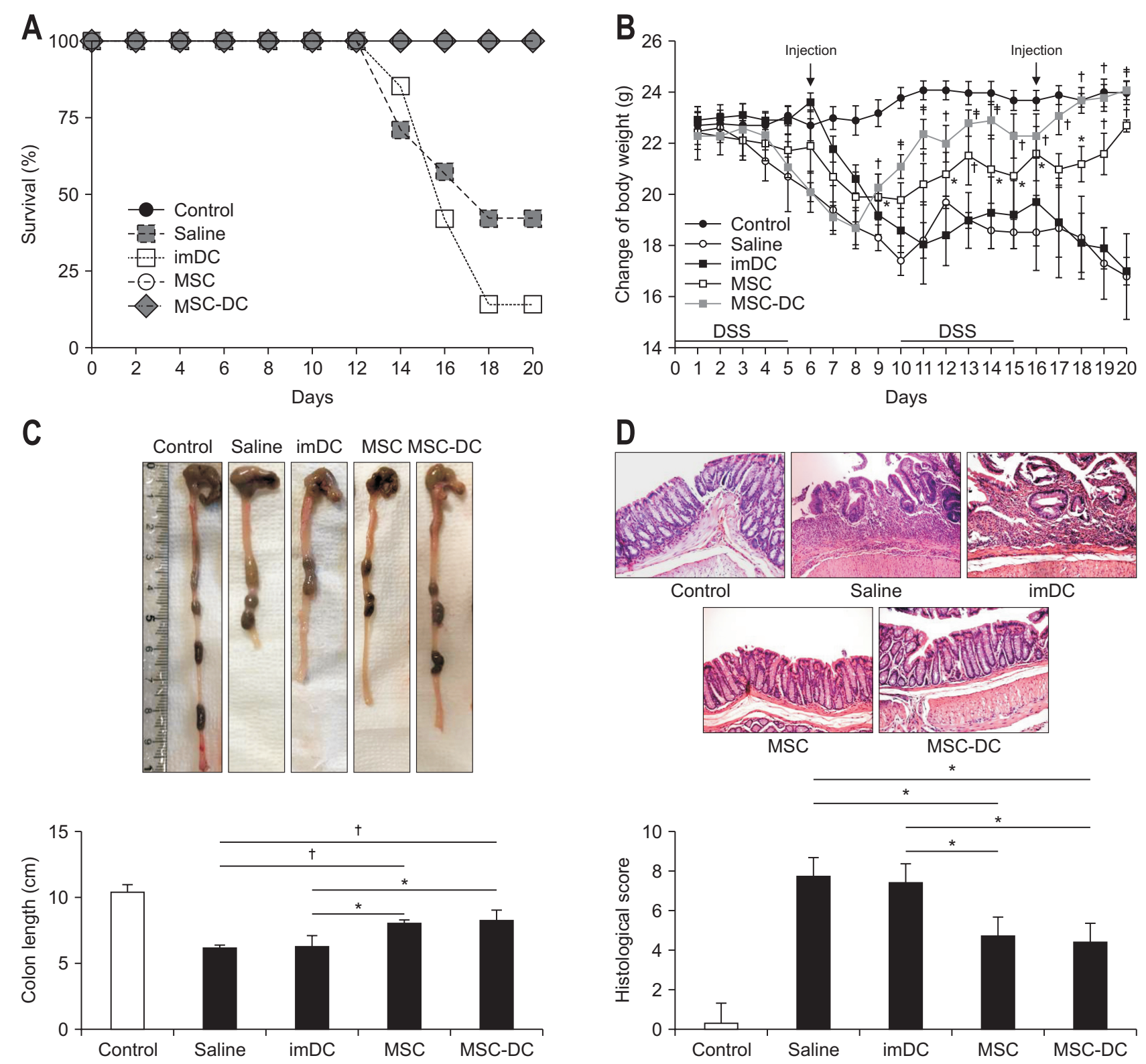

Fig. 4. MSC-DCs ameliorate dextran sodium sulfate (DSS)-induced chronic colitis in mice. Saline or $1 \times 10^{6}$ cells (imDCs, MSCs, and MSC-DCs) were administered by two intraperitoneal injections on days 6 and 16. (A) Survival rates of mice were analyzed (n=7). Injection with MSC-DCs significantly improved the survival rate. (B) The body weights of mice were recorded during the experiment $(\mathrm{n}=5)$. Treatment with MSCs and MSC-DCs significantly decreased the weight loss caused by DSS-induced colitis. (C) Colon length for each group ( $\mathrm{n}=5)$. MSC-DCs prevented the shortening of the colon length ( $p=0.003$ ). (D) Representative images of the colon tissue sections for histological examination (H\&E, $\times 20)$. The saline group had the highest inflammatory score; in contrast, the MSC-DCs group had the lowest score $(p=0.03)$. Statistical analysis was performed using a one-way analysis of variance.

MSCs, mesenchymal stem cells; DCs, dendritic cells; imDC, immature DCs. ${ }^{*} \mathrm{p}<0.05,{ }^{\dagger} \mathrm{p}<0.001$.

and IFN- $\gamma$ ), increased the expression of anti-inflammatory cytokines (IL-10 and TGF- $\beta$ ), and activated Treg cells, similar to in vitro results. These results demonstrate that MSC-DCs secreting anti-inflammatory cytokines (IL-10 and TGF- $\beta$ ) play a similar role as rDCs, resulting in the activation of Tregs. However, the precise mechanisms underlying the effect of MSCs on DC immunomodulation remain unclear. In this study, we did not analyze the changes in the DC phenotype of DSS-treated mice injected with cells (i.e., imDCs, MSCs, or MSC-DCs). Therefore, it is unclear at the present time whether the increase of Treg cells in the colon tissues of the MSC or MSC-DC injected groups correlates with suppression of host DCs by MSCs or MSC-DCs. Further studies are required to clarify whether MSCs or MSCDCs can suppress DCs in DSS-treated mice: first, whether either TGF- $\beta$ or IL-10 contributes to the differentiation of DCs into rDCs; second, how MSC-DCs interact with naïve T cells; and third, whether or not the injected MSC-DCs induce the host DCs to differentiate into rDCs. 
In conclusion, our results suggest that MSCs induce a change from immature and mature DC phenotype to rDC phenotype. MSC-DCs have similar functions to rDCs, thereby alleviating

Table 2. Colonic Histological Scoring System

\begin{tabular}{lcl}
\hline Histological feature & Score & \multicolumn{1}{c}{ Description } \\
\hline Epithelial damage & 0 & None \\
& 1 & 0\%-5\% loss of epithelium \\
2 & $5 \%-10 \%$ loss of epithelium \\
& 3 & Over 10\% loss of epithelium \\
Loss of crypts & 0 & None \\
& 1 & $0 \%-10 \%$ loss of crypts \\
& 2 & $10 \%-20 \%$ loss of crypts \\
Infiltration of & 3 & Over 20\% loss of crypts \\
inflammatory cells & 0 & None \\
& 1 & Mild (10\%) \\
& 2 & Moderate (25\%) \\
& 3 & Severe (40\%) \\
\hline
\end{tabular}

A

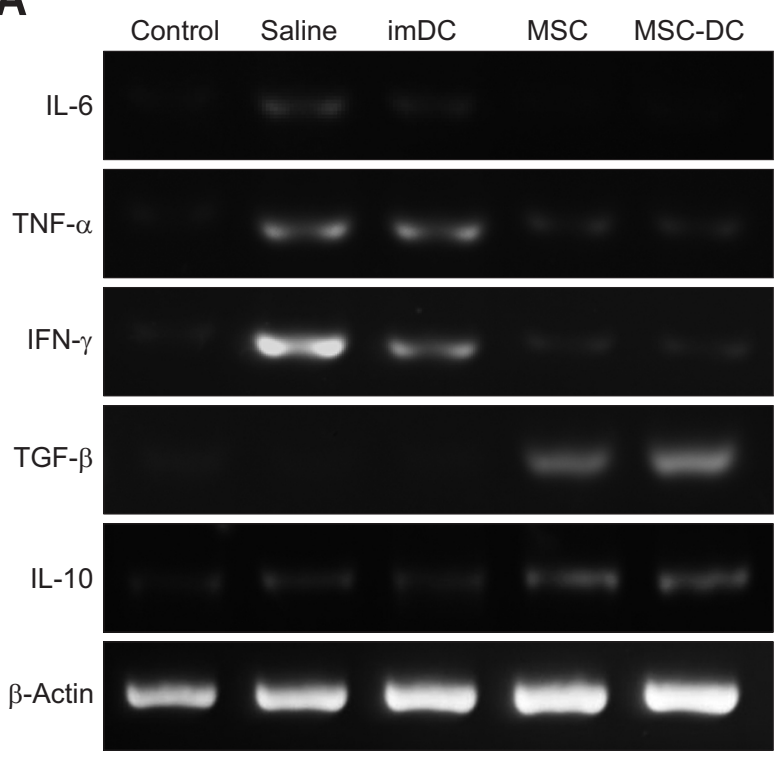

DSS-induced chronic colitis in vivo, similar to the role of MSCs in suppressing inflammation. Moreover, when co-cultured with splenocytes and injected into DSS-induced chronic colitis mice, MSC-DCs induced the activation of Tregs, which expressed Foxp3. Taken together, MSCs and MSC-DCs can also activate Tregs in vivo, and these Tregs can improve chronic colitis. In addition, our results also suggest that MSC-DCs or rDCs may potentially be used in the treatment of inflammatory diseases, such as IBD, similar to MSCs, which are utilized for various immunosuppressive treatments both in vivo and in vitro.

\section{CONFLICTS OF INTEREST}

No potential conflict of interest relevant to this article was reported.

\section{ACKNOWLEDGEMENTS}

This research was supported by a research grant from Yonsei University Wonju College of Medicine.

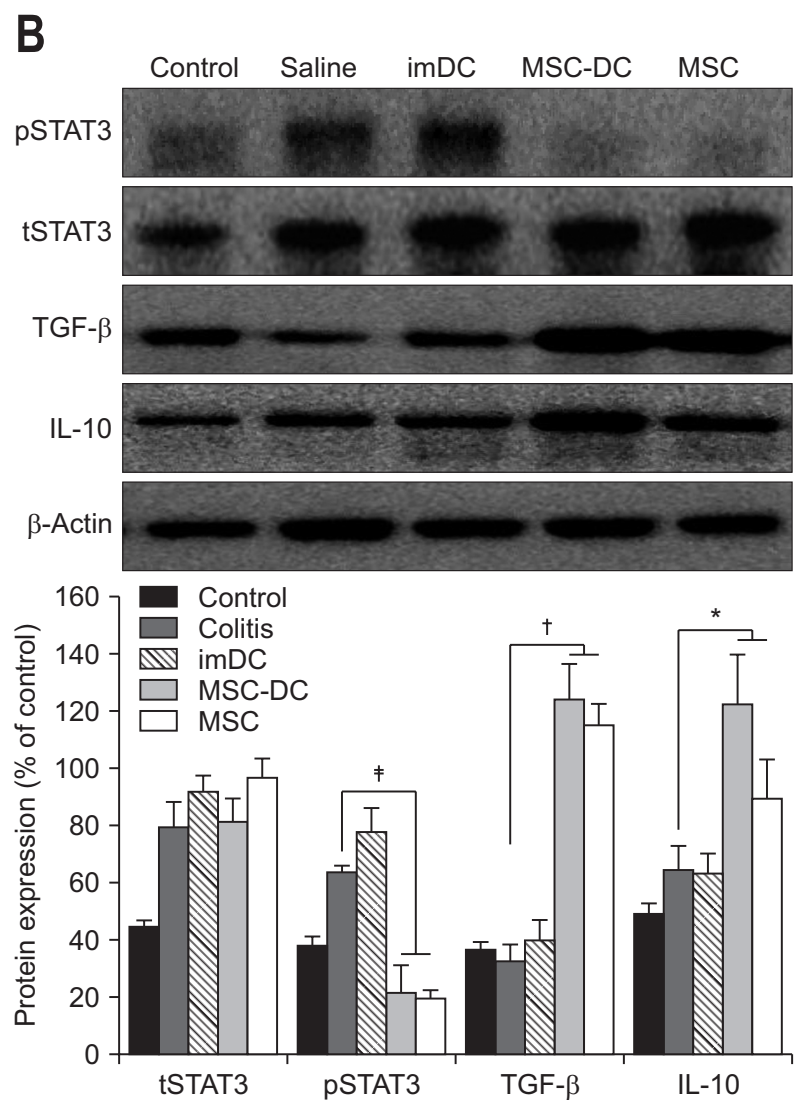

Fig. 5. MSC-DCs produce anti-inflammatory cytokines in dextran sodium sulfate (DSS)-induced chronic colitis mice. (A) Reverse transcriptionpolymerase chain reaction was performed to assess the mRNA levels of interleukin (IL)-6, tumor necrosis factor (TNF)- $\alpha$, interferon (IFN)- $\gamma$, IL-10, and transforming growth factor (TGF)- $\beta$. (B) Western blotting was performed to analyze the expression levels of total STAT3, phospho-STAT3, TGF- $\beta$, and IL- 10 . MSCs, mesenchymal stem cells; DCs, dendritic cells. ${ }^{*} \mathrm{p}<0.05,{ }^{\dagger} \mathrm{p}<0.001$, and ${ }^{\ddagger} \mathrm{p}<0.0001$. 

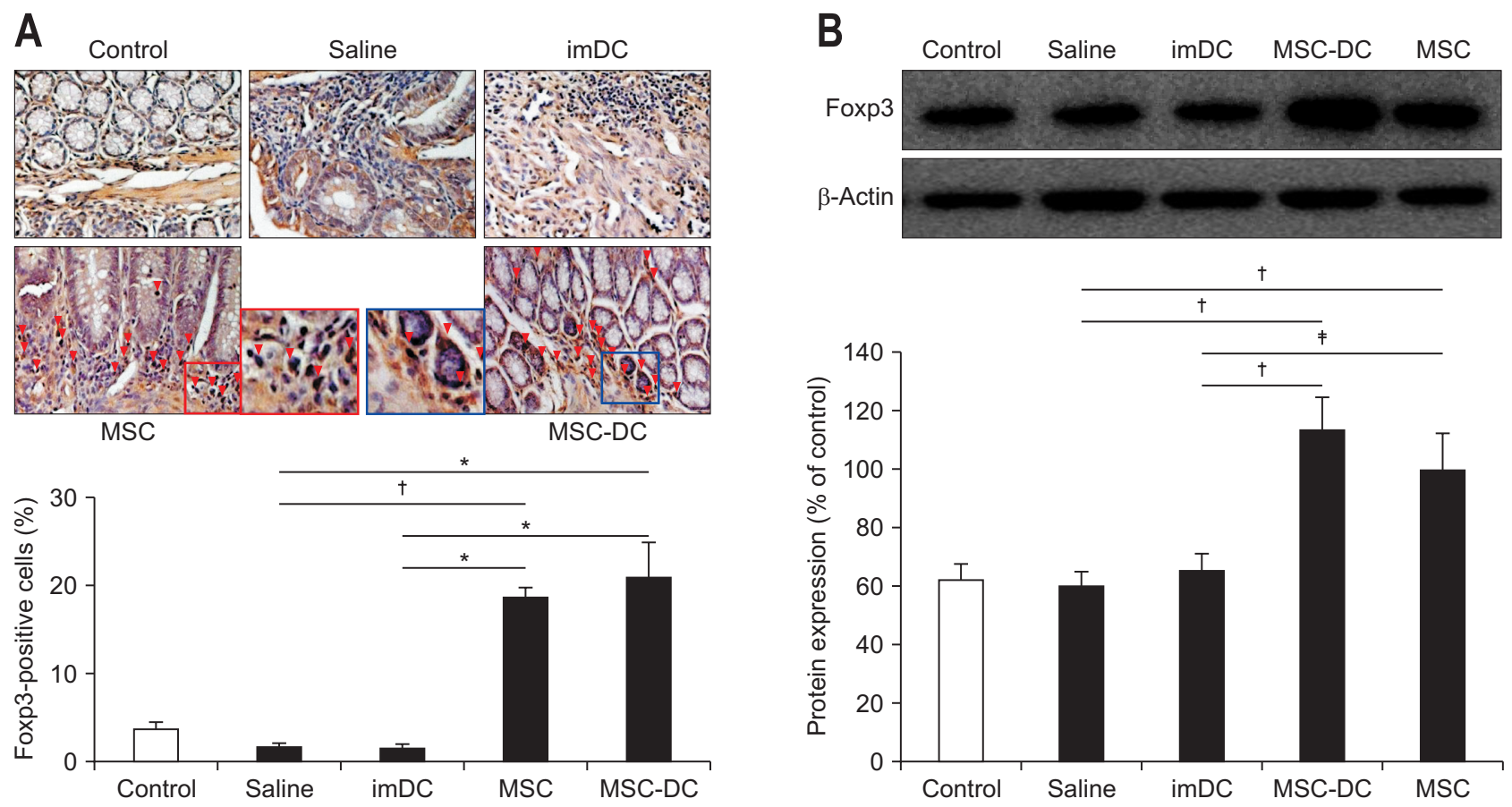

Fig. 6. Both mesenchymal stem cells (MSCs) and MSC-DCs induce regulatory T cell (Treg) differentiation in vivo. (A) Immunohistochemical staining $(\times 100)$ of Foxp $3^{+}$cells in the colon tissues. Representative images from one experiment are shown. Foxp $3^{+}$cells were counted from three random fields, with over 200 cells counted per field. (B) The number of Foxp $3^{+}$cells was expressed as a percentage of the total cell number. Foxp3 in the colon tissues was detected by Western blotting. Statistical analysis was performed using a one-way analysis of variance.

DC, dendritic cells; imDC, immature DCs. ${ }^{*} \mathrm{p}<0.05,{ }^{\dagger} \mathrm{p}<0.001$, and ${ }^{\ddagger} \mathrm{p}<0.0001$.

\section{REFERENCES}

1. Zhang YZ, Li YY. Inflammatory bowel disease: pathogenesis. World J Gastroenterol 2014;20:91-99.

2. Bouma G, Strober W. The immunological and genetic basis of inflammatory bowel disease. Nat Rev Immunol 2003;3:521-533.

3. Klinker MW, Wei CH. Mesenchymal stem cells in the treatment of inflammatory and autoimmune diseases in experimental animal models. World J Stem Cells 2015;7:556-567.

4. Duijvestein M, van den Brink GR, Hommes DW. Stem cells as potential novel therapeutic strategy for inflammatory bowel disease. J Crohns Colitis 2008;2:99-106.

5. Dalal J, Gandy K, Domen J. Role of mesenchymal stem cell therapy in Crohn's disease. Pediatr Res 2012;71(4 Pt 2):445-451.

6. Li W, Ren G, Huang Y, et al. Mesenchymal stem cells: a doubleedged sword in regulating immune responses. Cell Death Differ 2012;19:1505-1513.

7. Figueroa FE, Carrión F, Villanueva S, Khoury M. Mesenchymal stem cell treatment for autoimmune diseases: a critical review. Biol Res 2012;45:269-277.

8. Djouad F, Plence P, Bony C, et al. Immunosuppressive effect of mesenchymal stem cells favors tumor growth in allogeneic animals. Blood 2003;102:3837-3844.

9. Chen QQ, Yan L, Wang CZ, et al. Mesenchymal stem cells alleviate TNBS-induced colitis by modulating inflammatory and autoimmune responses. World J Gastroenterol 2013;19:4702-4717.
10. Lee HJ, Oh SH, Jang HW, et al. Long-term effects of bone marrow-derived mesenchymal stem cells in dextran sulfate sodiuminduced murine chronic colitis. Gut Liver 2016;10:412-419.

11. Liu WH, Liu JJ, Wu J, et al. Novel mechanism of inhibition of dendritic cells maturation by mesenchymal stem cells via interleukin-10 and the JAK1/STAT3 signaling pathway. PLoS One 2013;8:e55487.

12. Zhao ZG, Xu W, Sun L, et al. Immunomodulatory function of regulatory dendritic cells induced by mesenchymal stem cells. Immunol Invest 2012;41:183-198.

13. Zhang B, Liu R, Shi D, et al. Mesenchymal stem cells induce mature dendritic cells into a novel Jagged-2-dependent regulatory dendritic cell population. Blood 2009;113:46-57.

14. Bernhard H, Disis ML, Heimfeld S, Hand S, Gralow JR, Cheever MA. Generation of immunostimulatory dendritic cells from human CD34+ hematopoietic progenitor cells of the bone marrow and peripheral blood. Cancer Res 1995;55:1099-1104.

15. Banchereau J, Briere F, Caux C, et al. Immunobiology of dendritic cells. Annu Rev Immunol 2000;18:767-811.

16. Steinman RM, Hawiger D, Nussenzweig MC. Tolerogenic dendritic cells. Annu Rev Immunol 2003;21:685-711.

17. Mahnke K, Schmitt E, Bonifaz L, Enk AH, Jonuleit H. Immature, but not inactive: the tolerogenic function of immature dendritic cells. Immunol Cell Biol 2002;80:477-483.

18. Joffre O, Nolte MA, Spörri R, Reis e Sousa C. Inflammatory signals in dendritic cell activation and the induction of adaptive immu- 
nity. Immunol Rev 2009;227:234-247.

19. Bates J, Diehl L. Dendritic cells in IBD pathogenesis: an area of therapeutic opportunity? J Pathol 2014;232:112-120.

20. Chen L, Zhang W, Yue H, et al. Effects of human mesenchymal stem cells on the differentiation of dendritic cells from CD34+ cells. Stem Cells Dev 2007;16:719-731.

21. Spaggiari GM, Abdelrazik H, Becchetti F, Moretta L. MSCs inhibit monocyte-derived DC maturation and function by selectively interfering with the generation of immature DCs: central role of MSC-derived prostaglandin E2. Blood 2009;113:6576-6583.

22. Sato K, Yamashita N, Yamashita N, Baba M, Matsuyama T. Regulatory dendritic cells protect mice from murine acute graft-versushost disease and leukemia relapse. Immunity 2003;18:367-379.

23. Wood KJ, Bushell A, Hester J. Regulatory immune cells in transplantation. Nat Rev Immunol 2012;12:417-430.

24. Rayudu V, Raju AB. Effect of Triphala on dextran sulphate sodium-induced colitis in rats. Ayu 2014;35:333-338.

25. Zhu H, Guo ZK, Jiang XX, et al. A protocol for isolation and cul- ture of mesenchymal stem cells from mouse compact bone. Nat Protoc 2010;5:550-560.

26. Lin CS, Xin ZC, Dai J, Lue TF. Commonly used mesenchymal stem cell markers and tracking labels: limitations and challenges. Histol Histopathol 2013;28:1109-1116.

27. Levings MK, Bacchetta R, Schulz U, Roncarolo MG. The role of IL10 and TGF-beta in the differentiation and effector function of $\mathrm{T}$ regulatory cells. Int Arch Allergy Immunol 2002;129:263-276.

28. Wallet MA, Sen P, Tisch R. Immunoregulation of dendritic cells. Clin Med Res 2005;3:166-175.

29. Sato K, Yamashita N, Baba M, Matsuyama T. Modified myeloid dendritic cells act as regulatory dendritic cells to induce anergic and regulatory T cells. Blood 2003;101:3581-3589.

30. Chen W. Tregs in immunotherapy: opportunities and challenges. Immunotherapy 2011;3:911-914.

31. Chen ZM, O'Shaughnessy MJ, Gramaglia I, et al. IL-10 and TGFbeta induce alloreactive CD4+CD25- T cells to acquire regulatory cell function. Blood 2003;101:5076-5083. 